\title{
MULTIVARIATE RISK PROCESSES WITH INTERACTING INTENSITIES
}

\author{
NICOLE BÄUERLE, ${ }^{* * *}$ Universität Karlsruhe $(T H)$ \\ RUDOLF GRÜBEL, ${ }^{* * * * *}$ Leibniz Universität Hannover
}

\begin{abstract}
The classical models in risk theory consider a single type of claim. In the insurance business, however, several business lines with separate claim arrival processes appear naturally, and the individual claim processes may not be independent. We introduce a new class of models for such situations, where the underlying counting process is a multivariate continuous-time Markov chain of pure-birth type and the dependency of the components arises from the fact that the birth rate for a specific claim type may depend on the number of claims in the other component processes. Under certain conditions, we obtain a fluid limit, i.e. a functional law of large numbers for these processes. We also investigate the consequences of such results for questions of interest in insurance applications. Several specific subclasses of the general model are discussed in detail and the Cramér asymptotics of the ruin probabilities are derived in particular cases.
\end{abstract}

Keywords: Cramér asymptotic; fluid limit; Lundberg coefficient; multidimensional birth process; probability of ruin; risk reserve process; urn model

2000 Mathematics Subject Classification: Primary 60K99

Secondary 91B30

\section{Introduction}

Recently, in the insurance business, a tendency towards more advanced mathematical models can be observed. One reason for this development certainly is the discussion around the regulatory framework of Solvency II. An important topic in this discussion is still the problem of modelling dependencies. Whereas this question reduces to the concept of copulas in the case of multivariate random vectors, the situation is much more complicated if multivariate stochastic processes are involved. At present only a few attempts have been made to address the question of how to model dependent processes in a way that might be useful for insurance and finance applications. One possibility is the class of multivariate Lévy processes. For these, the dependence structure can be characterised by what is known as the Lévy copula of the process; see [4, p. 161]. Dependence properties of this class are further investigated in [3]. However, the disadvantage of this class is that — by definition — it does not show any dependence across time. When we restrict ourselves to counting processes, there are first suggestions for multidimensional models in [1], [14], and [19]. In [14] the authors constructed the vector of random numbers of events up to time $T$ by an ordinary copula and extracted the process by conditioning. Zocher [19] modelled the dependence by a random stochastic intensity vector

Received 23 April 2007; revision received 27 March 2008.

* Postal address: Institut für Stochastik, Universität Karlsruhe (TH), D-76128 Karlsruhe, Germany.

** Postal address: Institut für Mathematische Stochastik, Leibniz Universität Hannover, Postfach 6009, D-30167

Hannover, Germany. Email address: rgrubel@stochastik.uni-hannover.de

*** This work has been supported by the Deutsche Forschungsgemeinschaft (DFG). 
which was sampled once at the beginning, and in [1] the authors proposed a class of models where event points are produced by thinning and shifts from a homogeneous Poisson process. In the present paper we propose a model that avoids the reference to such an external mechanism by assuming instead that the event intensities of the components are interacting. We also go beyond the modelling of the counting process and investigate the behaviour of ruin probabilities in the corresponding risk reserve processes.

To be specific, suppose that we have an insurance company with different lines of business. In each individual line the difference of assets and liabilities can be modelled as a onedimensional risk reserve process. However, it makes sense not to assume that the business lines are independent. We propose to model the dependence by allowing the rate of arrivals of a claim of a specific type to depend on what happens in the other lines, specifically on the number of claims that have arrived in the individual components so far. For example, a large amount of claims in one business line may give rise to a higher or lower intensity in the other lines. Thus, intensities are interacting. Models like this have been considered in connection with credit risk.

The paper is organised as follows. In Section 2 we supply the formal definitions of the counting processes and the risk reserve processes that are relevant for our models. For the counting processes, we also give some typical examples with simulated data; these and the corresponding illustrations are meant to provide an intuitive access, complementing the formal definitions, to the problems and questions that we consider in the later sections. In Section 3 we look at the general dependence structure and give conditions under which the counting processes are associated. In Section 4 we show that, again under certain conditions, a fluid limit can be obtained, and we derive some properties of the limit. We also derive such results for the associated risk reserve processes; in Section 5 we discuss these limits in connection with insurance applications. We show that, in the context of actuarial applications, a very dangerous situation may arise if the fluid limit itself is stochastic. In this case, if the premium rate is chosen according to the expected value principle, the ruin probability may not converge to 0 if the initial reserve tends to $\infty$. In Section 6 we investigate a class of models with repelling intensities. We look at the behaviour of ruin probabilities for the corresponding multivariate risk reserve processes and obtain the Cramér asymptotics. In Section 7 we consider processes with attracting intensities and again derive the Cramér asymptotics. It turns out that the Lundberg exponent is the same as for the corresponding model with independent claims. In Sections 6 and 7 we find a connection to the classical urn models of Pólya and Eggenberger, and Friedman, respectively.

\section{The general model}

We assume that we have $d$ business lines, labelled $j=1, \ldots, d$. The counting process for business line $j$ is denoted by $N_{j}$, so that $N_{j}(t)$ gives the number of claims of type $j$ that have arrived by time $t$. We further assume that at most one such event, of whatever type, happens at any given time and that the number of events in any finite interval is finite with probability 1 . This means that the paths of $N_{j}$ are continuous from the right and have left limits (càdlàg), and that the jumps all have size 1 . Let $N=(N(t))_{t \geq 0}$, where $N(t)=\left(N_{1}(t), \ldots, N_{d}(t)\right)$ is the vector process that consists of the $d$ one-dimensional counting processes $\left(N_{j}(t)\right)_{t \geq 0}, j=1 \ldots, d$. The state space for the process $N$ is $E:=\mathbb{N}_{0}^{d}$ and it has càdlàg paths. Our basic assumption is that $N$ is a time-homogeneous Markov chain; note that the components may not have the Markov property individually. This differs from the approach in [1], where the authors started with the assumption that the components are Poisson processes with constant intensity. 
(a)

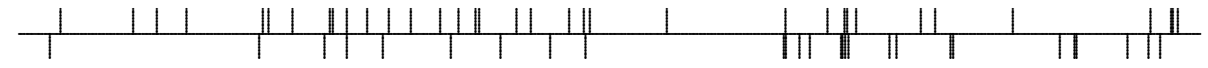

(b)

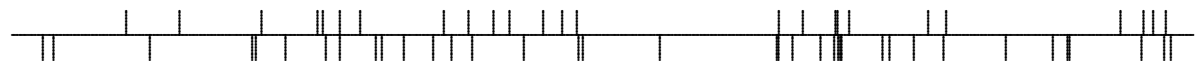

(c)

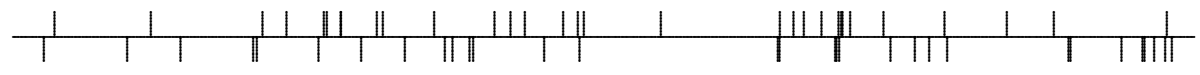

(d)

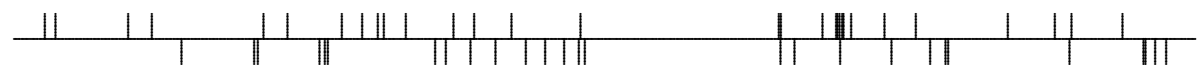

Figure 1: Paths of four two-dimensional claim arrival processes.

Later we will rescale the state space; $E$ and its rescaled versions are regarded as subsets of the $d$-dimensional upper right orthant $\mathbb{R}_{+}^{d}$. We set $\|x\|:=\left|x_{1}\right|+\cdots+\left|x_{d}\right|$ for all $x=$ $\left(x_{1}, \ldots, x_{d}\right) \in \mathbb{R}^{d}$; in particular, $\|N(t)\|$ is the total number of events up to and including time $t$. In view of our general assumption that at any given time at most one event can take place, the jumps of $N$ are from the set $B:=\left\{e_{1}, \ldots, e_{d}\right\}$, where $e_{j} \in E$ has $j$ th component 1 and all other components equal to 0 . Hence, $N=(N(t))_{t \geq 0}$ may be regarded as a multidimensional pure-birth process and its stochastic structure (distribution) is specified by the distribution of the number $N(0)$ of counts at time 0 and the rates for the different jumps, i.e. with suitable functions $\beta_{j}, j=1, \ldots, d$,

$$
\mathrm{P}\left[N(t+h)=k+e_{j} \mid N(t)=k\right]=h \beta_{j}(k)+o(h) \quad \text { as } h \rightarrow 0
$$

for all $k=\left(k_{1}, \ldots, k_{d}\right) \in E$. We write $\beta$ for the $\mathbb{R}_{+}^{d}$-valued function with component functions $\beta_{1}, \ldots, \beta_{d}$. We may assume that $\|\beta(k)\|>0$ for all $k \in E$, since the process would 'freeze' at states $k \in E$ with $\beta(k)=0$. We will further find it convenient to assume that the birth-rate functions are bounded in the sense that

$$
\eta:=\sup _{k \in E}\|\beta(k)\|<\infty
$$

An important consequence is

$$
\mathrm{P}\left[N_{j}(t)<\infty \text { for all } t \geq 0, j=1, \ldots, d\right]=1,
$$

i.e. such an assumption prevents 'explosion' in finite time.

This general framework contains several interesting subclasses. We illustrate these in dimension $d=2$, where we now present four special models that will be used throughout the paper. Explicit examples for each of these, with artificial data, are given in Figure 1. In Figure 1 the horizontal lines represent time and the arrival of a claim is marked by a short vertical line, pointing upwards for claims of the first type and downwards otherwise.

Of course, if the functions $\beta_{1}$ and $\beta_{2}$ are constant then the components are independent Poisson processes. Figure 1(a) shows a realisation of the independent model with $\beta_{1}=\beta_{2} \equiv \frac{1}{2}$. In Figure 1(b) we have

$$
\beta_{1}\left(k_{1}, k_{2}\right)=\frac{1+k_{1}}{2+k_{1}+k_{2}}, \quad \beta_{2}\left(k_{1}, k_{2}\right)=\frac{1+k_{2}}{2+k_{1}+k_{2}},
$$


and in Figure 1(c) we have

$$
\beta_{1}\left(k_{1}, k_{2}\right)=\frac{1+k_{2}}{2+k_{1}+k_{2}}, \quad \beta_{2}\left(k_{1}, k_{2}\right)=\frac{1+k_{1}}{2+k_{1}+k_{2}},
$$

respectively. In Figure 1(b) an increase in the number of claims of a particular type increases the probability of the next claim being of the same type, which could be regarded as a case with repelling intensities. In Figure 1(c) a large number of claims in one component increases the likelihood for the next claim to be of the other type, which could be regarded as a case with attracting intensities.

In Figure 1(a)-(c) the counting process can be constructed from a background Poisson process with constant rate by a random mechanism that channels the arrivals to the different claim types, with the probabilities depending on the number of claims in the different business lines so far. For independent components, i.e. in Figure 1(a), the channeling step may simply consist of tossing a coin. In Figure 1(d) we consider a situation where deletion of the background events is possible:

$$
\beta_{1}\left(k_{1}, k_{2}\right) \equiv 1, \quad \beta_{2}\left(k_{1}, k_{2}\right)= \begin{cases}1 & \text { if } k_{2}<k_{1}, \\ 0 & \text { otherwise, }\end{cases}
$$

claims that the coin would designate to the second type are deleted as soon as the total number of claims in the second component reaches the total number of claims in the first component.

A useful tool in the study of continuous-time Markov chains, such as the present multivariate claim counting process $N=(N(t))_{t \geq 0}$, is the embedded jump chain $X=(X(n))_{n \in \mathbb{N}_{0}}$, where the paths of $X$ consist of the sequence of states that $N$ visits successively. With $N$ as above, $X$ moves from $k \in E$ to $k+e_{i}$ with probability

$$
p_{k, k+e_{i}}=\frac{\beta_{i}(k)}{\|\beta(k)\|}, \quad i=1, \ldots, d .
$$

Figure 2 shows the graphs of the (interpolated) paths for the jump chains associated with the data in Figure 1. These are also the graphs of the function $t \mapsto N(t)$; for comparison, the diagonals are included.

While this is not our main concern in the present paper, we note that such plots may serve as a graphical tool to detect dependencies between the component processes, in much the same way as the well-known scatterplots do for random vectors. Indeed, that there might be a major difference in the models generating (b) and (c) respectively, seems easier to see in Figure 2 than in Figure 1.

In the first three of the four examples the sum of the coordinate intensities is constant, so we may reconstruct $N$ from $X$ by $N(t):=X(L(t))$, where $L=(L(t))_{t \geq 0}$ is a Poisson process with constant rate $\|\beta\|$, and $X$ and $L$ are independent. More generally, in all cases where (1) is satisfied, $N$ can be constructed from a Markov chain $X$ in discrete time and a Poisson process $L$ with rate $\eta$ in the above manner. We now use the uniformised chain $X$, which has transition probabilities

$$
p_{k, k+e_{i}}=\frac{\beta_{i}(k)}{\eta}, \quad i=1, \ldots, d,
$$

complemented by $p_{k, k}=1-\eta^{-1}\|\beta(k)\|$. This simple device, of 'obtaining $N$ by running $X$ according to a Poisson clock $L$ ', often makes it easy to transfer results from $X$ to $N$. This technique is known as uniformisation and can also be used to simulate the processes. 


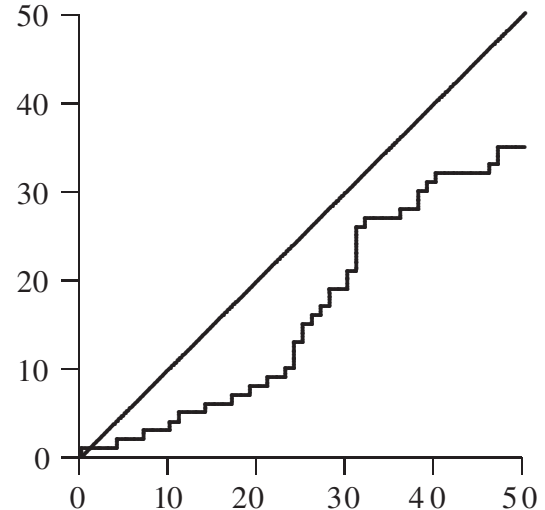

(a)

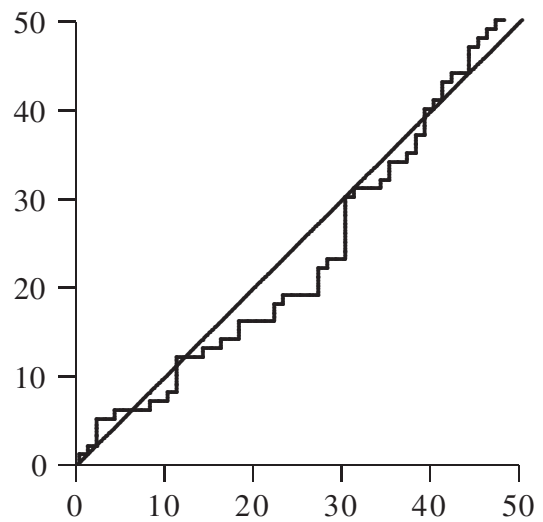

(c)

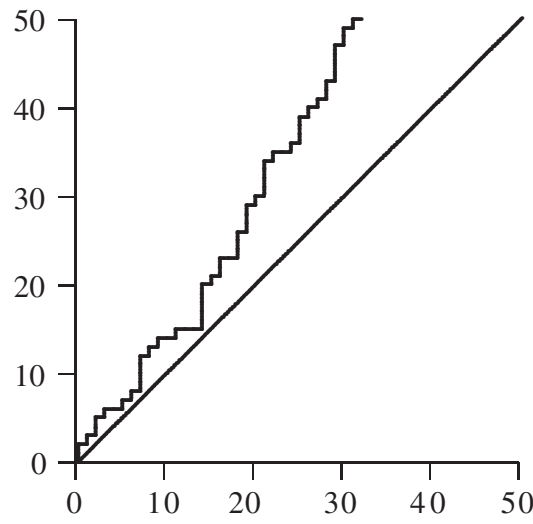

(b)

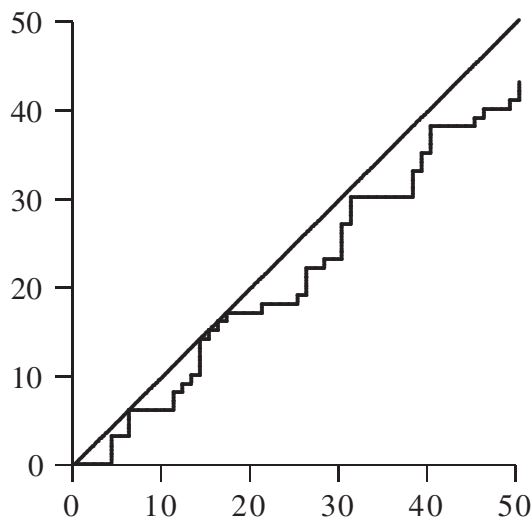

(d)

FIGURE 2: Path graphs of the jump chains.

On the basis of these multivariate counting processes we can now construct the corresponding multivariate risk reserve processes. We will generally assume that, for each of the $d$ business lines, we have a sequence $\left(U_{j, k}\right)_{k \in \mathbb{N}}$ of independent nonnegative random variables, all with the same distribution $Q_{j}, j=1, \ldots, d$; we also assume that the random variables are independent across the coordinates. Furthermore, we have a premium income rate $c_{j}$ for the $j$ th business line and a fixed initial capital $R_{j}(0)$. The multivariate risk reserve process $R=\left(R_{t}\right)_{t \geq 0}$ is then defined by $R(t)=\left(R_{1}(t), \ldots, R_{d}(t)\right)$, with

$$
R_{j}(t):=R_{j}(0)+c_{j} t-\sum_{k=1}^{N_{j}(t)} U_{j, k} \quad \text { for all } t \geq 0, j \in\{1, \ldots, d\} .
$$

For the risk reserve processes, we will generally assume that $R_{j}(0)$ is deterministic and that $N_{j}(0)=0$. One of the most important quantities associated with $R$ is the probability of ruin, first in the individual components, with

$$
\psi_{j}(u):=\mathrm{P}\left[\inf _{t \geq 0} R_{j}(t)<0 \mid R_{j}(0)=u\right]
$$


being the probability of ruin of business line $j$, but also, for the sum $R_{0}:=\sum_{j=1}^{d} R_{j}$ of the component processes,

$$
\psi_{0}(u):=\mathrm{P}\left[\inf _{t \geq 0} R_{0}(t)<0 \mid R_{0}(0)=u\right] .
$$

A central issue in this setup is the dependence of the ruin probabilities on the type of interaction between the claim intensities. The standard one-dimensional theory does not apply to the component processes, since the components $N_{j}$ of the multivariate counting process $N$ are in general not constant rate Poisson processes (neither are they renewal processes, which would be required in the Sparre-Anderson generalisation of the standard model). The sum process $R_{0}$, however, may well be a standard one-dimensional risk reserve process, with premium income rate $c_{0}:=c_{1}+\cdots+c_{d}$ and claim arrival process $\|N\|$. This is the case, for example, if $\|\beta\|$ is a constant function, as in the models underlying parts (a)-(c) of Figures 1 and 2, and if, additionally, the $Q_{j}$ s do not depend on $j$. Moreover, in case of (a), or more generally in $d$ dimensions with $\beta_{j} \equiv \lambda_{j}$ for some $\lambda_{1}, \ldots, \lambda_{d} \geq 0, R_{0}$ is again a standard one-dimensional risk reserve process. The associated claim arrival rate is $\lambda_{0}:=\lambda_{1}+\cdots+\lambda_{d}$ and the claim size distribution is given by $\lambda_{0}^{-1}\left(\lambda_{1} Q_{1}+\cdots+\lambda_{d} Q_{d}\right)$; note that the $Q_{j}$ s may be different in this situation. Of course, from our present point of view, this latter case is not very interesting as there is no interaction between the components.

In the next two sections we deal with the general model and obtain results on stochastic order properties and the asymptotic behaviour under fluid scaling. We will then investigate models that contain the above repelling and attracting examples (b) and (c), respectively, as special cases, where we also address the question of the influence of the interaction on the ruin probabilities.

\section{Dependence structure}

It is quite obvious that the component processes of $N$ are in general dependent. Moreover, it is possible to identify cases where the components show some kind of positive dependence. There are numerous concepts for dependence and we will restrict ourselves here to the notion of association: a random vector $X=\left(X_{1}, \ldots, X_{d}\right)$ is called (positively) associated if $\operatorname{cov}(f(X), g(X)) \geq 0$ for all increasing functions $f, g: \mathbb{R}^{d} \rightarrow \mathbb{R}$. Here and in the following we use the coordinatewise order on $\mathbb{R}^{d}$, and monotonicity refers to this order. For vectorvalued functions, such as the intensity function $\beta$ of $N$, increasing means that all the component functions are increasing in the above sense.

The concept of association can be extended to stochastic processes (see Chapter 3.7 of [17] for the following definitions). A stochastic process $X=(X(t))_{t \geq 0}$ with values in $\mathbb{R}^{d}$ is said to be associated if $\left(X\left(t_{1}\right), \ldots, X\left(t_{n}\right)\right)$ is associated (as an $n d$-dimensional vector) for all $0 \leq t_{1}<t_{2}<\cdots<t_{n}$ and all $n \in \mathbb{N}$. Note that the property of association remains valid under any permutation of the random variables. In order to state our result, we need a second important property: a Markov process $X$ is said to be stochastically monotone if

$$
x \mapsto \mathrm{E}[f(X(t)) \mid X(s)=x]
$$

is an increasing function for all increasing $f: \mathbb{R}^{d} \rightarrow \mathbb{R}$ and for all $0 \leq s<t$.

There are many results about stochastic monotonicity for stochastic processes; however, we have not found the following version.

Lemma 1. Let $N$ be a multidimensional pure-birth process, as defined in Section 2, with intensity function $\beta: E \rightarrow \mathbb{R}_{+}^{d}$. If $\beta$ is increasing then $N$ is stochastically monotone. 
Proof. The transition probabilities of the corresponding uniformised chain $(X(n))_{n \in \mathbb{N}}$ are given by

$$
\mathrm{P}\left[X_{n+1}=k+e_{j} \mid X_{n}=k\right]=\frac{\beta_{j}(k)}{\eta}, \quad j=1, \ldots, d .
$$

With probability $1-\eta^{-1}\|\beta(k)\|$, no state change occurs; see also the discussion in Section 2 . It is easy to see that the assumption on $\beta$ implies that $X=(X(n))_{n \in \mathbb{N}_{0}}$ is stochastically monotone, and this transfers to $N=(N(t))_{t \geq 0}$ in a straightforward manner since the distributions of $N$ can be regarded as Poisson mixtures of the distributions of $X$.

The following is the main result of this section.

Theorem 1. If $\beta$ is increasing then $N$ is associated.

Proof. We apply Theorem 2.14 of [11]. Note that $N$ is a Feller process and that it is stochastically monotone by Lemma 1 . Thus, it remains to check that

$$
\mathcal{A}(f g) \geq g \mathscr{A} f+f \mathscr{A} g
$$

for all increasing functions $f, g: \mathbb{N}^{d} \rightarrow \mathbb{R}$. Here $\mathcal{A}$ denotes the generator of $N$, which is given by

$$
\mathcal{A} f(k)=\sum_{j=1}^{d}\left(f\left(k+e_{j}\right)-f(k)\right) \beta_{j}(k) .
$$

An easy calculation shows that $\mathcal{A}(f g) \geq g \mathcal{A} f+f \mathscr{A} g$ if and only if

$$
\sum_{j=1}^{d} \beta_{j}(k)\left(f\left(k+e_{j}\right)-f(k)\right)\left(g\left(k+e_{j}\right)-g(k)\right) \geq 0 .
$$

Since $f$ and $g$ are increasing, the proof is complete.

\section{Fluid limits}

In this section we derive the fluid limits of the multidimensional claim counting and risk reserve processes that we introduced in Section 2. The fluid model is the result of a functional law of large numbers and reveals the general path behaviour of the stochastic processes. We rescale time and state space by the same factor $\gamma>0$ and then let $\gamma$ tend to $\infty$; see, e.g. Section 5.4 of [18] for a general reference. Formally, and taking into account the fact that the rescaling should not force the starting values to become 0 , we can define, for each $\gamma>0$, an operator $\Psi_{\gamma}$ that maps functions $f: \mathbb{R}_{+} \rightarrow \mathbb{R}^{d}$ to functions $\Psi_{\gamma}(f): \mathbb{R}_{+} \rightarrow \mathbb{R}^{d}$ by

$$
\Psi_{\gamma}(f)(t):=f(0)+\frac{1}{\gamma}(f(\gamma t)-f(0)) \text { for all } t \geq 0 .
$$

With the counting processes $N$ and the risk reserve processes $R$ defined in Section 2, we set $N^{\gamma}:=\Psi_{\gamma}(N)$ and $R^{\gamma}:=\Psi_{\gamma}(R)$. In particular, $N^{\gamma}$ is the process with jump intensity $\gamma \beta$ that starts at the same initial state as $N$ and has jumps of size $\gamma^{-1}$. Our main result, Theorem 2 , holds for counting processes which have an arbitrary (deterministic) value $N(0) \in \mathbb{N}^{d}$ at 0 . However, when we deal with the risk reserve processes, we will always assume that $N(0)=0$. Thus, we can write the components of $N^{\gamma}$ and $R^{\gamma}$ as

$$
N_{j}^{\gamma}(t)=N_{j}(0)+\frac{1}{\gamma}\left(N_{j}(\gamma t)-N_{j}(0)\right)
$$


and

$$
R_{j}^{\gamma}(t)=R_{j}(0)+c_{j} t-\frac{1}{\gamma} \sum_{k=1}^{N_{j}(\gamma t)} U_{j, k}
$$

for all $t \geq 0$ and $j=1, \ldots, d$.

We are interested in the potential limits of the processes $N^{\gamma}$ and $R^{\gamma}$ as $\gamma \rightarrow \infty$. We need two auxiliary results. For the first of these, we introduce the processes $\left(M_{j}(t)\right)_{t \geq 0}$ with

$$
M_{j}(t):=N_{j}(t)-N_{j}(0)-\int_{0}^{t} \beta_{j}(N(s)) \mathrm{d} s \quad \text { for } j=1, \ldots, d \text { and all } t \geq 0,
$$

and $(M(t))_{t \geq 0}$ with $M(t):=\left(M_{1}(t), \ldots, M_{d}(t)\right)$ for all $t \geq 0$. The following lemma is a standard fact; see, e.g. [5, Theorem 26.14] .

Lemma 2. The process $M$ is a d-dimensional martingale with respect to $\left(\mathcal{F}_{t}\right)_{t \geq 0}$.

Obviously, we can write (in vector notation)

$$
N(t)=N(0)+M(t)+\int_{0}^{t} \beta(N(s)) \mathrm{d} s .
$$

Rescaling time and space as described above we obtain

$$
N^{\gamma}(t)=N(0)+M^{\gamma}(t)+\int_{0}^{t} \beta(N(\gamma s)) \mathrm{d} s
$$

with $M^{\gamma}:=\Psi_{\gamma}(M)$. The processes $N^{\gamma}, \gamma>0$, are defined on a common probability space $(\Omega, \mathcal{F}, \mathrm{P})$ and each $N^{\gamma}$ is a random element with values in $D^{d}[0, \infty)$, the space of $\mathbb{R}^{d}$-valued functions on $[0, \infty)$ that are càdlàg. We endow $D^{d}[0, \infty)$ with the Skorokhod topology. The symbol $\stackrel{\text { D }}{\rightarrow}$ ' denotes the convergence in distribution in this space; this will appear in the theorem below. Our second auxiliary result, however, deals with convergence in probability. For continuous limit functions, convergence in $D^{d}[0, \infty)$ with respect to the Skorokhod topology is known to be equivalent to uniform convergence on compact sets. Hence, in order to prove that a sequence $\left(X_{n}\right)_{n \in \mathbb{N}}$ of random elements of $D^{d}[0, \infty)$ converges in probability to 0 as $n \rightarrow \infty$, it is enough to show that

$$
\lim _{n \rightarrow \infty} \mathrm{P}\left[\sup _{0 \leq s \leq t}\left|X_{n}(s)\right|>\epsilon\right]=0 \text { for all } \epsilon>0 \text { and } t>0 .
$$

Lemma 3. The process $M^{\gamma}$ converges to 0 in probability as $\gamma \rightarrow \infty$.

Proof. First choose a line $j$ and define the stopping time

$$
\tau_{n}^{j}:=\inf \left\{t \geq 0|| M_{j}^{\gamma}(t) \mid \geq n\right\} .
$$

Then $\left(M_{j}^{\gamma}\left(t \wedge \tau_{n}^{j}\right)\right)_{t \geq 0}$ is a square-integrable martingale, since it is bounded. Using Fatou's lemma and denoting the quadratic variation of the process $X$ by $[X]$, we obtain

$$
\begin{aligned}
\mathrm{E}\left[M_{j}^{\gamma}(t)^{2}\right] & \leq \liminf _{n \rightarrow \infty} \mathrm{E}\left[M_{j}^{\gamma}\left(t \wedge \tau_{n}^{j}\right)^{2}\right] \\
& \leq \liminf _{n \rightarrow \infty} \mathrm{E}\left[\left[M_{j}^{\gamma}\right]\left(t \wedge \tau_{n}^{j}\right)\right] \\
& \leq \frac{1}{\gamma^{2}} \mathrm{E}\left[\text { number of jumps of } M_{j}^{\gamma} \text { in }[0, t]\right] \\
& \leq \frac{\eta t}{\gamma} .
\end{aligned}
$$


Hence, by Doob's inequality,

$$
\mathrm{E}\left[\sup _{0 \leq s \leq t} M_{j}^{\gamma}(s)^{2}\right] \leq 4 \mathrm{E}\left[M_{j}^{\gamma}(t)^{2}\right] \leq \frac{4 \eta t}{\gamma} .
$$

The statement of the lemma now follows on using the above criterion for convergence in probability to 0 together with Markov's inequality.

We write $\lceil x\rceil$ for the vector with components $\left\lceil x_{j}\right\rceil, j=1, \ldots, d$, for $x=\left(x_{1}, \ldots, x_{d}\right) \in$ $\mathbb{R}_{+}^{d}$. In $\mathbb{R}^{d}$ we have the probability simplex

$$
W_{d}:=\left\{\left(x_{1}, \ldots, x_{d}\right) \in \mathbb{R}_{+}^{d}: x_{1}+\cdots+x_{d}=1\right\},
$$

to which we transfer the $(d-1)$-dimensional Lebesgue measure in the canonical fashion. The following is the main theorem of this section. In it we require that the intensity function $\beta$ converges to a function $\tilde{\beta}: W_{d} \rightarrow \mathbb{R}^{d}$ along rays in the sense that, for almost all $x \in W_{d}$ and all sequences $\left(x_{n}\right)_{n \in \mathbb{N}} \subset \mathbb{R}_{+}^{d}$,

$$
\lim _{n \rightarrow \infty}\left\|x_{n}\right\|=\infty, \quad \lim _{n \rightarrow \infty} \frac{x_{n}}{\left\|x_{n}\right\|}=x \Longrightarrow \lim _{n \rightarrow \infty} \beta\left(\left\lceil x_{n}\right\rceil\right)=\tilde{\beta}(x) .
$$

We extend $\tilde{\beta}$ to $\mathbb{R}_{+}^{d} \backslash\{0\}$ by $\tilde{\beta}(x)=\tilde{\beta}\left(\|x\|^{-1} x\right)$. With $x, y \in \mathbb{R}^{d}$, we denote by $x \circ y=$ $\left(x_{1} y_{1}, \ldots, x_{d} y_{d}\right)$ the componentwise product. Let $c=\left(c_{1} \ldots, c_{d}\right)$ be the vector of the premium income rates.

Theorem 2. Suppose that $\beta$ satisfies (1) and (6) and that the distribution $Q_{j}$ of the claims of type $j$ has finite second moment and mean $\mu_{j}$. Then every sequence $(\gamma(k))_{k \in \mathbb{N}} \subset(0, \infty)$ with $\gamma(k) \rightarrow \infty$ has a subsequence $(\gamma(k(l)))_{l \in \mathbb{N}}$ such that

$$
N^{\gamma(k(l))} \stackrel{\mathrm{D}}{\rightarrow} \phi \quad \text { and } \quad R^{\gamma(k(l))} \stackrel{\mathrm{D}}{\rightarrow} r \quad \text { as } l \rightarrow \infty .
$$

Furthermore, the paths of the limit processes $\phi$ and $r$ are almost surely absolutely continuous, and they satisfy

$$
\begin{gathered}
\phi(t)=\phi(0)+\int_{0}^{t} \tilde{\beta}(\phi(s)-\phi(0)) \mathrm{d} s, \\
r(t)=r(0)+c t-\mu \circ \phi(t) .
\end{gathered}
$$

Proof. In order to be able to use (6), the total number $\|N(t)\|$ of claims up to time $t$ needs to converge to $\infty$ with probability 1 as $t \rightarrow \infty$. For this, we note that any ball in the $\|\cdot\|$-norm with finite radius will eventually be left and not be visited again since

$$
\inf \{\|\beta(k)\|: k \in E,\|k\| \leq M\}>0 \text { for all } M<\infty,
$$

which in turn follows from our general assumption that $\|\beta(k)\|>0$ for all $k \in E$ and from the fact that the infimum is over a finite set.

We now show that the family $\left\{N^{\gamma}: \gamma \geq 1\right\}$ is tight. For this, we check the usual sufficient conditions (see, e.g. [10, p. 251]):

$$
\begin{gathered}
\lim _{m \rightarrow \infty} \sup _{\gamma \geq 1} \mathrm{P}\left[\left\|N^{\gamma}(t)\right\| \geq m\right]=0 \quad \text { for all } t \geq 0, \\
\lim _{\delta \rightarrow 0} \limsup \sup _{\gamma \rightarrow \infty} \mathrm{E}\left[\min \left\{1,\left\|N^{\gamma}(\tau+\delta)-N^{\gamma}(\tau)\right\|\right\}\right]=0 .
\end{gathered}
$$


In (10) the supremum is over the set of stopping times $\tau$ with respect to the filtration generated by $N$. The uniformisation implies that the total number $\|N(t)-N(0)\|$ of jumps in the time interval $(0, t]$ is bounded from above by a random variable that has a Poisson distribution with parameter $\eta t$. Hence, Markov's inequality implies that, for all $m$,

$$
\begin{aligned}
\mathrm{P}\left[\left\|N^{\gamma}(t)\right\| \geq m\right] & \leq \frac{1}{m^{2}} \mathrm{E}\left[\left\|N^{\gamma}(t)\right\|^{2}\right] \\
& \leq \frac{3}{m^{2}}\left(\mathrm{E}\left[\|N(0)\|^{2}\right]+\mathrm{E}\left[\left\|N^{\gamma}(t)-N(0)\right\|^{2}\right]\right) \\
& \leq \frac{3}{m^{2}}\left(\mathrm{E}\left[\|N(0)\|^{2}\right]+\frac{1}{\gamma^{2}} \mathrm{E}\left[\|N(\gamma t)-N(0)\|^{2}\right]\right) \\
& \leq \frac{3}{m^{2}}\left(\mathrm{E}\left[\|N(0)\|^{2}\right]+\frac{1}{\gamma^{2}}\left(\eta \gamma t+(\eta \gamma t)^{2}\right)\right),
\end{aligned}
$$

which shows that (9) is true. For (10), we condition on the pre- $\tau \sigma$-field and use the strong Markov property of $N$ to obtain

$$
\mathrm{E}\left[\min \left\{1,\left\|N^{\gamma}(\tau+\delta)-N^{\gamma}(\tau)\right\|\right\}\right] \leq \delta \eta,
$$

which directly implies the desired statement. Thus, we obtain the existence of converging subsequences.

Now suppose that we have a converging sequence $N^{\gamma(k)}$ with $\gamma(k) \rightarrow \infty$ as $k \rightarrow \infty$ and denote the limit of $N^{\gamma(k)}$ by $\phi$. Using the Skorokhod representation theorem, we may assume that this convergence is almost sure instead of in distribution. Let the basic $\omega$ (which we suppress in the notation) be such that we have the convergence in $D^{d}[0, \infty)$. By the argument given at the beginning of the proof we may also assume that $N^{\gamma(k)}(t) \rightarrow \infty$ as $t \rightarrow \infty$. Using property (6), we now obtain, for each $s>0$,

$$
\begin{aligned}
\lim _{k \rightarrow \infty} \beta(N(\gamma(k) s)) & =\tilde{\beta}\left(\lim _{k \rightarrow \infty} \frac{\left(N^{\gamma(k)}(s)-N(0)\right) \gamma(k)+N(0)}{\left\|\left(N^{\gamma(k)}(s)-N(0)\right) \gamma(k)+N(0)\right\|}\right) \\
& =\tilde{\beta}\left(\lim _{k \rightarrow \infty} \frac{\left(N^{\gamma(k)}(s)-N(0)\right)+N(0) / \gamma(k)}{\left\|\left(N^{\gamma(k)}(s)-N(0)\right)+N(0) / \gamma(k)\right\|}\right) \\
& =\tilde{\beta}\left(\frac{\phi(s)-\phi(0)}{\|\phi(s)-\phi(0)\|}\right) \\
& =\tilde{\beta}(\phi(s)-\phi(0)) .
\end{aligned}
$$

Because of (1), we can apply dominated convergence, which yields

$$
\lim _{k \rightarrow \infty} \int_{0}^{t} \beta(N(\gamma(k) s)) \mathrm{d} s=\int_{0}^{t} \tilde{\beta}(\phi(s)-\phi(0)) \mathrm{d} s .
$$

Combining this with (5) and Lemma 3, we obtain the integral equation for $\phi$. The absolute continuity of $t \mapsto \phi(t)$ is an immediate consequence of this equation.

For the analysis of the risk reserve process, we first note that the limit result for the counting processes implies that $\tilde{R}^{\gamma}$ with

$$
\tilde{R}_{j}^{\gamma}(t):=R_{j}(0)+c_{j} t-\mu_{j} N_{j}^{\gamma}(t)
$$


converges in distribution to $r$ along suitably chosen subsequences. It is therefore enough to show that the difference $\tilde{R}^{\gamma}-R^{\gamma}$ converges to 0 in probability as $\gamma \rightarrow \infty$, which in turn would follow from

$$
\lim _{\gamma \rightarrow \infty} \mathrm{P}\left[\sup _{0 \leq s \leq t}\left|\frac{1}{\gamma} \sum_{k=1}^{N_{j}(\gamma t)}\left(U_{j, k}-\mu_{j}\right)\right|>\epsilon\right]=0
$$

for all $\epsilon>0, t>0$, and $j=1, \ldots, d$. Conditionally on $N$, the partial sums $\sum_{k=1}^{n}\left(U_{j, k}-\right.$ $\left.\mu_{j}\right), n \in \mathbb{N}$, are a martingale and $N_{j}(\gamma t), t>0$, is an increasing family of deterministic, and hence bounded, stopping times. The optional sampling theorem together with Doob's inequality therefore lead to

$$
\begin{aligned}
\mathrm{P}\left[\sup _{0 \leq s \leq t}\left|\sum_{k=1}^{N_{j}(\gamma t)}\left(U_{j, k}-\mu_{j}\right)\right|>\epsilon \mid N\right] & \leq \frac{4}{\epsilon^{2}} \mathrm{E}\left[\left(\sum_{k=1}^{N_{j}(\gamma t)}\left(U_{j, k}-\mu_{j}\right)\right)^{2} \mid N\right] \\
& =\frac{4}{\epsilon^{2}} N_{j}(\gamma t) \mathrm{E}\left[U_{j, 1}^{2}\right] .
\end{aligned}
$$

In view of $\mathrm{E}\left[N_{j}(\gamma t)\right] \leq \eta \gamma t$ we now obtain (11) by taking expectations. This completes the proof of the theorem.

We note in passing that assumption (1) can be replaced by the weaker condition

$$
\mathrm{E}\left[N^{\gamma}(t)\right]=O_{t}(\gamma) \text { and } \mathrm{E}\left[N^{\gamma}(t)\right]^{2}=O_{t}\left(\gamma^{2}\right) \text { for all } t \geq 0 \text { as } \gamma \rightarrow \infty
$$

where the index $t$ means that the relevant constants may depend on $t$.

The family of solutions to (7) and (8) is called the fluid limit of the counting process or risk reserve process, respectively. The fluid limit for the counting process has some interesting properties, which we collect in the following result.

Theorem 3. (a) If $\tilde{\beta}$ is locally Lipschitz continuous on $W_{d}$ then the solution to (7) is unique up to a set of measure 0 whenever $\|\phi(0)\|>0$.

(b) The paths $t \mapsto \phi(t)$ of the fluid limit are nondecreasing and can grow at most linearly.

Proof. Part (a) is an immediate consequence of the Phragmen-Lindelöf theorem, a standard result from the theory of differential equations.

For part (b), note that $\tilde{\beta}$ cannot take negative values, which implies that $\phi$ is nondecreasing. From (1) and (6), we find that $\tilde{\beta}$ is bounded; hence, the growth restriction follows from (7).

If the fluid limit for $N$ is a unique and deterministic function $\phi$, then $N^{\gamma}$ converges to $\phi$ in probability, a result that may be interpreted as a functional weak law of large numbers. Of course, the analogous statement then also holds for the risk reserve processes, as $r$ is simply a function of $\phi$.

\section{Applications of fluid limits}

Naturally, some information will be lost when passing to the fluid model, as will be demonstrated in Subsection 5.1. We then show that, nevertheless, the results of the previous section are useful in the insurance context, especially in connection with questions concerning the long-term behaviour of the processes. 


\subsection{Limitations of the fluid model}

If $\beta_{j}$ depends on $k_{j}$ only then there is no interaction and the component processes are independent. In two dimensions, for example, with $\beta_{1}=\beta_{2} \equiv \frac{1}{2}$, the counting process $N$ is simply a pair of independent Poisson processes with rates $\frac{1}{2}$. The fluid limit $\phi$ is deterministic and consists of the one path $t \mapsto(t / 2, t / 2)$.

Now suppose, still with $d=2$, that

$$
\beta_{1}\left(k_{1}, k_{2}\right)=\left\{\begin{array}{ll}
1 & \text { if } k_{2}=k_{1}+1, \\
\frac{1}{2} & \text { if } k_{2}=k_{1}, \\
0 & \text { otherwise }
\end{array} \quad \text { and } \quad \beta_{2}\left(k_{1}, k_{2}\right)= \begin{cases}1 & \text { if } k_{1}=k_{2}+1 \\
\frac{1}{2} & \text { if } k_{2}=k_{1} \\
0 & \text { otherwise }\end{cases}\right.
$$

This is the tightest coupling between the component processes that is compatible with the assumption that at most one event occurs at any given time. The claim numbers in the two components never differ by more than 1 . In this model the times between successive claims (of whatever type) are independent and exponentially distributed with mean 1. Consequently, if we start with zero claims in both components then $N^{\gamma}$ will converge with probability 1 to the deterministic fluid limit $\phi(t)=(t / 2, t / 2)$.

These two examples show that the dependence structure may not survive the passage to the fluid limit. We will see in Section 6, however, that, for cases with repelling intensities, such as in Figure 1(b), the dependence of the components may lead to random fluid limits, where the distributions of $\phi$ and $r$ depend on the claim intensities.

\subsection{Proportion of claim arrivals of different types}

A natural question in the context of multivariate counting processes concerns the average number of claims in the different business lines or, for example, the proportion of claims in the long run for two fixed business lines. Such questions can easily be answered with the help of fluid limits. By ' $\rightarrow$ ' we denote convergence in probability.

Theorem 4. Suppose that the conditions of Theorem 2 hold and that $\tilde{\beta}$ is locally Lipschitz continuous. Let $\phi$ denote the fluid limit. Then, for all $i, j \in\{1, \ldots, d\}$, we have, for $t \rightarrow \infty$,

$$
\frac{N_{i}(t)}{t} \stackrel{\mathrm{P}}{\rightarrow} \phi_{i}(1)-\phi_{i}(0), \quad \frac{N_{i}(t)}{N_{j}(t)} \stackrel{\mathrm{P}}{\rightarrow} \frac{\phi_{i}(1)-\phi_{i}(0)}{\phi_{j}(1)-\phi_{j}(0)} .
$$

Proof. Note that since $\tilde{\beta}$ is locally Lipschitz continuous, Theorem 3(a) implies that the solution to (7) is unique and that we have $N^{\gamma} \stackrel{\mathrm{D}}{\rightarrow} \phi$ for $\gamma \rightarrow \infty$. Now by (4) we obtain

$$
\frac{N_{i}(\gamma)}{\gamma}=N_{i}^{\gamma}(1)-N_{i}(0)+\frac{1}{\gamma} N_{i}(0)
$$

Thus, Theorem 2 yields

$$
\frac{N_{i}(\gamma)}{\gamma} \stackrel{\mathrm{D}}{\rightarrow} \phi_{i}(1)-\phi_{i}(0),
$$

and since the limit is a constant, we also have convergence in probability. The second part of the assertion is a consequence of the first part and the continuous mapping theorem. 


\subsection{Insurability}

In this subsection we discuss the question of choosing adequate premium rates for insurance lines with claims arriving according to the model in Section 2. As in Section 2, we assume that the claim sizes in each business line $j$ are nonnegative, independent, and identically distributed random variables $U_{j, k}, k \in \mathbb{N}$, all with the same distribution $Q_{j}$, that claims are stochastically independent across the coordinates $j=1, \ldots, d$, and that the family of claim sizes is independent of the arrival process $N$. (Some of these assumptions can be weakened.) We recall that $\psi_{j}(u)$ denotes the probability of eventual ruin in business line $j$, if the initial capital for that line is $u$.

A first task, which is rather simple in the classical ruin setting, is to choose the premium rate $c_{j}$ for business line $j$ such that $\psi_{j}(u)<1$ for all $u \geq 0$. In the interacting context this question is more complicated. The following is a first observation.

Lemma 4. We have $\psi_{j}(u)=1$ for some $u \geq 0$ if and only if $\psi_{j}(u)=1$ for all $u \geq 0$.

Proof. Obviously the statement for all $u \geq 0$ implies the one for a fixed $u \geq 0$. Now fix $u \geq 0$ and suppose that $\psi_{j}(u)=1$. The monotonicity of the probability measure yields $\psi_{j}\left(u^{\prime}\right)=1$ for all $0 \leq u^{\prime} \leq u$. Now let $u^{\prime}>u$ and suppose that $\psi_{j}\left(u^{\prime}\right)<1$. However, since

$$
\left\{N_{j}\left(\left(u^{\prime}-u\right) c_{j}^{-1}\right)=0\right\} \supset\left\{L\left(\left(u^{\prime}-u\right) c_{j}^{-1}\right)-L(0)=0\right\}
$$

and the probability of the latter event is $\exp \left(-\left(u^{\prime}-u\right) c_{j}^{-1} \eta\right)>0$, this would imply that $\psi_{j}(u)<1$, which is a contradiction.

The fluid limit can now serve as a tool for deciding whether $\psi_{j}(u)=1$. We say that the fluid limit defined by (7) and (8) is not insurable for business line $j$ if, with $r(0) \equiv 0$, every path of the fluid limit has the property that there exists a $t_{0}>0$ such that $r_{j}\left(t_{0}\right)<0$.

Theorem 5. If the fluid limit is not insurable for business line $j$ then $\psi_{j}(u)=1$ for all $u \geq 0$.

Proof. According to Lemma 4 it suffices to show that $\psi_{j}(0)=1$. In Theorem 2 choose a convergent subsequence $\left(N^{\gamma(k)}, R^{\gamma(k)}\right)_{k \in \mathbb{N}}$ with $\lim _{k \rightarrow \infty} \gamma(k)=\infty$. By Skorokhod's theorem, the process can be constructed on the same probability space such that the convergence is almost everywhere. Since the limit is continuous with probability 1 , the convergence is uniform on compact sets. Now fix an arbitrary $\omega \in \Omega$ and suppose that $R^{\gamma(k)}(t)(\omega) \rightarrow r(t)$ on an interval $[0, T]$, where $r$ is a solution to (8). Let $t_{0} \in[0, T]$ ( $T$ has to be large enough) be such that $r_{j}\left(t_{0}\right)<0$ and choose $k$ large enough to obtain $R_{j}^{\gamma(k)}\left(t_{0}\right)(\omega)<-\varepsilon$ with $r_{j}\left(t_{0}\right)<-\varepsilon<0$. This implies that $R_{j}\left(\gamma(k) t_{0}\right)(\omega)<-\varepsilon \gamma(k)<0$. Thus, for this trajectory, we have ruin at time $t_{0} \gamma(k)$ or earlier.

Moreover, a very dangerous situation may arise whenever the fluid limit is stochastic, a situation which we will encounter in Section 6. To illustrate the situation, let us look at component $j$ with initial reserve $u \geq 0$. Obviously, we have, for all $\gamma \geq 1$ and $t>0$,

$$
\psi_{j}(u) \geq \psi_{j}(u \gamma) \geq \mathrm{P}\left[R_{j}^{\gamma}(t)<0 \mid R_{j}(0)=u\right] .
$$

Assuming that we can apply Theorem 2 and letting $\gamma \rightarrow \infty$ this yields

$$
\psi_{j}(u) \geq \mathrm{P}\left[r_{j}(t)<0 \mid r_{j}(0)=u\right] \text { for all } t \geq 0,
$$

where the distribution function of $r_{j}(t)$ is continuous in 0 . Note that we have $r_{j}(t)=r_{j}(0)$ $+c_{j} t-\mu_{j} \phi_{j}(t)$. In what follows we assume that the fluid limit is stochastic and that $\phi_{j}(t) / t \stackrel{\mathrm{D}}{=} \xi$ 
independent of $t$, where $\xi$ has a finite expectation $\mathrm{E}[\xi]$ (these conditions are fulfilled for the models in Section 6) and where ' $\stackrel{\text { D }}{=}$ denotes equality in distribution. Moreover, we assume that the insurance company calculates the premium rate according to the expected value principle and sets $c_{j}:=\mu_{j} \mathrm{E}[\xi]+\delta$ for some $\delta>0$. Then we obtain, with $t \rightarrow \infty$,

$$
\psi_{j}(u) \geq \mathrm{P}\left[\delta<\mu_{j}(\xi-\mathrm{E}[\xi])\right] .
$$

Note that the probability on the right-hand side does not depend on the initial reserve $u$ anymore and that it may well be positive if $\delta$ is too small. This means that we cannot obtain a Cramér asymptotic here; even worse, the ruin probability does not tend to 0 if $u$ tends to $\infty$. Bearing this in mind, a dependence between claim arrivals may be more dangerous than the case of heavy-tailed claims, for example, where a Cramér asymptotic does not exist either, but the ruin probability at least goes to 0 for $u$ tending to $\infty$. The risk in such a situation can only be managed by choosing a premium rate much higher than the expected cost rate.

\section{Models with repelling intensities}

The second of our basic examples has intensity function

$$
\beta_{1}\left(k_{1}, k_{2}\right)=\frac{1+k_{1}}{2+k_{1}+k_{2}}, \quad \beta_{2}\left(k_{1}, k_{2}\right)=\frac{1+k_{2}}{2+k_{1}+k_{2}} .
$$

It describes a situation where an increasing proportion of claims of one type leads to an increasing rate of arrival of claims of the same type. This intensity function satisfies condition (6) with

$$
\tilde{\beta}_{1}\left(x_{1}, x_{2}\right)=\frac{x_{1}}{x_{1}+x_{2}} \quad \text { and } \quad \tilde{\beta}_{2}\left(x_{1}, x_{2}\right)=\frac{x_{2}}{x_{1}+x_{2}} \quad \text { for all } x_{1}, x_{2} \in \mathbb{R}_{+}^{2} \backslash\{(0,0)\} .
$$

For simplicity, in this section we assume that the counting processes start at 0 . Then the associated fluid limit equation, (7), has a whole family $\left\{\phi_{\alpha}: 0 \leq \alpha \leq 1\right\}$ of solutions given by

$$
\phi_{\alpha}(t)=(\alpha t,(1-\alpha) t) \text { for all } t \geq 0 .
$$

This raises the question of what the long-term behaviour of the two-dimensional counting process $(N(t))_{t \geq 0}$ with the above intensity function is. In the examples given in Subsection 5.1 there was a single deterministic solution to (7), which implies that the limit distribution is degenerate. In such cases Theorem 2 gives a full description of the asymptotic behaviour. In cases where the fluid limit may be random, however, Theorem 2 would need to be augmented by statements concerning the distribution of $\phi$. We now carry this out for a class of models that contains the above as a special case.

For a multivariate counting process with intensity function (2), the embedded jump chain $X=(X(n))_{n \in \mathbb{N}_{0}}$ can be related to the classical Pólya-Eggenberger urn model: at time 0, we have one red ball and one blue ball. In the step from time $n$ to time $n+1$ we choose one of the (then $n+2$ ) balls uniformly at random and put it back into the urn, together with another ball of the same colour; $X_{1}(n)$ is the number of red balls in the urn after $n$ steps. It is known that the proportion $Z_{n}$ of red balls at time $n$ converges almost surely to a random variable $Z_{\infty}$ and that $Z_{\infty}$ is uniformly distributed on the unit interval; see, e.g. [8, Section 6.3.3] or [6, p. 243]. In view of the fact that $\beta_{1}+\beta_{2}$ is constant we can interpret the counting process as $X$ being run by a clock that is a constant rate Poisson process, as explained in Section 2, so that

$$
\frac{1}{t} N(t) \rightarrow\left(\begin{array}{c}
Z_{\infty} \\
1-Z_{\infty}
\end{array}\right) \quad \text { almost surely as } t \rightarrow \infty
$$


with $Z_{\infty}$ as above. This exemplifies a situation where the fluid limit is not deterministic, i.e. the randomness does not disappear at the present linear scaling.

It is well known that the Pólya-Eggenberger urn model arises as an $h$-transform of the random walk on $\mathbb{N}_{0} \times \mathbb{N}_{0}$ that moves from $(i, j)$ to either $(i+1, j)$ or $(i, j+1)$, where both these transitions have probability $\frac{1}{2}$. In fact, the bounded and nonnegative harmonic functions $h$ associated with this random walk can be written as

$$
h(i, j)=\int_{[0,1]} x^{i}(1-x)^{j} v(\mathrm{~d} x) \quad \text { for all }(i, j) \in E
$$

for some probability measure $v$ on the unit interval; see, e.g. [9, p. 394ff] or [16, Section 6]. For example, if $v$ is the beta distribution with parameters $\alpha_{1}$ and $\alpha_{2}$ then we obtain the transition probabilities

$$
\begin{aligned}
& p_{(i, j),(i+1, j)}=\frac{h(i+1, j)}{2 h(i, j)}=\frac{\alpha_{1}+i}{\alpha_{1}+\alpha_{2}+i+j}, \\
& p_{(i, j),(i, j+1)}=\frac{h(i, j+1)}{2 h(i, j)}=\frac{\alpha_{2}+j}{\alpha_{1}+\alpha_{2}+i+j},
\end{aligned}
$$

which contain (2) as a special case if the Poisson clock has rate 1.

Generalising this to $d$ dimensions, we start with the symmetric 'north-east' random walk, which has transition probabilities

$$
p_{k, k+e_{j}}=\frac{1}{d} \quad \text { for all } k=\left(k_{1}, \ldots, k_{d}\right) \in E, j=1, \ldots, d,
$$

and consider the $h$-transform associated with the harmonic function

$$
h(k)=\int_{W_{d}} x^{k} v(\mathrm{~d} x) \quad \text { for all } k \in E,
$$

where $v$ is some probability measure on the $d$-dimensional probability simplex $W_{d}$, and where we have used the abbreviation $x^{k}=x_{1}^{k_{1}} \cdots x_{d}^{k_{d}}$ for $x=\left(x_{1}, \ldots, x_{d}\right) \in \mathbb{R}^{d}$ and $k=$ $\left(k_{1}, \ldots, k_{d}\right) \in E$. If $v$ is concentrated on some $z=\left(z_{1}, \ldots, z_{d}\right) \in W_{d}$ then this leads to a north-east random walk with transition probabilities

$$
p_{k, k+e_{j}}^{(h)}=z_{j} \quad \text { for all } k=\left(k_{1}, \ldots, k_{d}\right) \in E, j=1, \ldots, d,
$$

so that we may interpret the general case as a mixture of such 'biased' random walks. A straightforward calculation shows that, for general $v$, the mixture continues to be a Markov chain, but that it will in general no longer be a random walk, and that its transition probabilities are

$$
p_{k, k+e_{j}}^{(h)}=\frac{\mathrm{E}\left[Z^{k+e_{j}}\right]}{\mathrm{E}\left[Z^{k}\right]} \quad \text { for all } k \in E, j=1, \ldots, d,
$$

where $Z$ is a random vector with distribution $v$. This provides the embedded jump chain $X$ from which we again obtain a multivariate counting process $N$ by using a one-dimensional Poisson process $L$ with constant intensity $\lambda$ as a random clock.

Alternatively, we can regard these models as a special class of mixed Poisson processes. The following construction of such processes is adapted to our purposes. For a given dimension $d$ and rate parameter $\lambda>0$, let $Z$ be a random variable with values in $W_{d}$ and distribution $v$, 
and let $L=(L(t))_{t \geq 0}$ be a one-dimensional Poisson process with constant intensity $\lambda$. Given $Z=\left(z_{1}, \ldots, z_{d}\right)$, we construct the $d$-dimensional counting process $N=\left(N_{1}, \ldots, N_{d}\right)$ by independently assigning each of the events of $L$ to the $j$ th component with probability $z_{j}$.

Putting this together, we obtain the following result. The second half is obvious from the construction of $N$; note that we even have almost sure convergence of $N^{(\gamma)}$ to $\phi$ as $\gamma \rightarrow \infty$.

Theorem 6. The multivariate counting process $N$ associated with $\lambda$ and $v$ in the above construction is a time-homogeneous Markov chain with intensities

$$
\beta_{j}(k)=\lambda \frac{\mathrm{E}\left[Z^{k+e_{j}}\right]}{\mathrm{E}\left[Z^{k}\right]} \text { for all } k \in E, j=1, \ldots, d,
$$

where $Z$ is a random vector with distribution $v$. Moreover, the fluid limit $\phi$ for $N$ is of the form

$$
\phi(t)=t \lambda Z \quad \text { for all } t \geq 0 .
$$

We regard $N$ as a directionally mixed Poisson process with mixing distribution $v$ and base rate $\lambda$. This distinguishes these models within the greater class of all mixed Poisson processes, which in general will not be homogeneous in time. For a thorough study of general mixed multivariate Poisson processes, we refer the reader to [20], which also contains a result similar to the first half of Theorem 6, with a detailed (and different) proof.

We now turn to the associated risk reserve processes. In the classical one-dimensional setting the parameters of the standard model consist of the claim arrival rate $\lambda$, the premium income rate $c$, and the claim size distribution $Q$. We assume that $Q$ is not a point mass (we consider the case of constant claim sizes as being degenerate), that

$$
H(t):=\int \mathrm{e}^{t x} Q(\mathrm{~d} x)<\infty \text { for some } t>0,
$$

and that

$$
\lim _{t \uparrow t_{0}} H(t)=\infty \quad \text { with } t_{0}:=\sup \{t>0: H(t)<\infty\} .
$$

Then the mean $\mu:=\int x Q(\mathrm{~d} x)$ associated with $Q$ is finite and, for $c>\lambda \mu$, there is a unique solution $\kappa=\kappa(\lambda, c, Q)>0$ of the equation

$$
H(t)=1+\frac{c t}{\lambda} .
$$

The ruin probability $\psi(u)$ then decreases asymptotically at an exponential rate as a function of the initial capital $u$; more precisely, we have the following Lundberg bound and Cramér limit:

$$
\psi(u) \leq \mathrm{e}^{-\kappa u} \quad \text { for all } u>0, \quad \lim _{u \rightarrow \infty} \frac{1}{u} \log \psi(u)=-\kappa
$$

(see, e.g. [15, Section 5.4.1]). The rate parameter $\kappa$ is known as the Lundberg exponent or adjustment coefficient.

In the multivariate case we have, as explained in Section 2, an initial capital $u_{j}$, a premium income rate $c_{j}$, and a claim size distribution $Q_{j}$ for the $j$ th business line, $j=1, \ldots, d$. In the following result we obtain the Lundberg exponent for the global ruin probability $\psi_{0}$ (which depends on $u_{1}, \ldots, u_{d}$ via $u:=u_{1}+\cdots+u_{d}$ ) in the case that the underlying multivariate counting process is a directionally mixed Poisson process. In it $\operatorname{supp}(v)$ denotes the support of the measure $v$, and we write $\langle x, y\rangle=\sum_{j=1}^{d} x_{j} y_{j}$ for the inner product of the vectors $x=$ $\left(x_{1}, \ldots, x_{d}\right) \in \mathbb{R}^{d}$ and $y=\left(y_{1}, \ldots, y_{d}\right) \in \mathbb{R}^{d}$. 
Theorem 7. Let $N$ be a directionally mixed Poisson process with mixing distribution $v$ and base rate $\lambda$, and let $R$ be the associated risk reserve process with nonnegative premium income rates $c_{1}, \ldots, c_{d}$ and claim size distributions $Q_{1}, \ldots, Q_{d}$. We assume that the claim size distributions are nondegenerate and that they satisfy (12) and (13). Suppose further that

$$
\|c\|>\lambda \sup \{\langle z, \mu\rangle: z \in \operatorname{supp}(v)\}
$$

where $c=\left(c_{1}, \ldots, c_{d}\right), \mu=\left(\mu_{1}, \ldots, \mu_{d}\right)$, and $\mu_{j}=\int x Q_{j}(\mathrm{~d} x), j=1, \ldots, d$. Then the global ruin function $\psi_{0}$ associated with the sum $R_{0}$ of the components of $R$ satisfies

$$
\psi_{0}(u) \leq \exp \left(-\kappa_{0} u\right) \quad \text { for all } u>0, \quad \lim _{u \rightarrow \infty} \frac{1}{u} \log \psi_{0}(u)=-\kappa_{0},
$$

where the Lundberg exponent $\kappa_{0}$ is given by

$$
\kappa_{0}=\inf \{\kappa(z): z \in \operatorname{supp}(\nu)\}
$$

and $\kappa(z)$ is the Lundberg exponent for the one-dimensional model with premium income rate $\|c\|$, claim arrival intensity $\lambda$, and claim size distribution $\sum_{j=1}^{d} z_{j} Q_{j}$.

Proof. Let $A \subset W_{d}$ be the support of $v$, and let $H_{j}$ be the moment generating function associated with $Q_{j}$, finite on $\left(-\infty, t_{0}^{j}\right)$. For each $z=\left(z_{1}, \ldots, z_{d}\right) \in A$ and with $Q_{z}:=\sum_{j=1}^{d} z_{j} Q_{j}$, the function

$$
t \mapsto \Psi(z, t):=\int \mathrm{e}^{t x} Q_{z}(\mathrm{~d} x)-1-\frac{\|c\| t}{\lambda}
$$

is differentiable, has the value 0 for $t=0$, and approaches $\infty$ as $t \rightarrow t(z)$ with $t(z):=$ $\min \left\{t_{0}^{j}: z_{j} \neq 0, j=1, \ldots, d\right\}$. Furthermore, its derivative at 0 is strictly negative in view of assumption (15) and

$$
\left.\frac{\partial}{\partial t} \int \mathrm{e}^{t x} Q_{z}(\mathrm{~d} x)\right|_{t=0}=\sum_{j=1}^{d} z_{j} \int x Q_{j}(\mathrm{~d} x)=\langle z, \mu\rangle,
$$

and, similarly, using the general assumption that the claim distributions are not degenerate, this function is strictly convex. This establishes the existence and uniqueness of a positive root $\kappa(z)$ for each $z \in W_{d}$. We now claim that $z \mapsto \kappa(z)$ is continuous. This follows with the implicit function theorem if we can show that

$$
\frac{\partial}{\partial t} \Psi(z, t) \neq 0 \quad \text { whenever } t>0 \text { and } \Psi(z, t)=0
$$

In fact,

$$
\sum_{j=1}^{d} z_{j} H_{j}^{\prime}(t)-\frac{\|c\|}{\lambda}>0 \text { if } t>0 \text { and } \sum_{j=1}^{d} z_{j} H_{j}(t)=1+\frac{\|c\| t}{\lambda},
$$

in view of the strict convexity of $t \mapsto \int \mathrm{e}^{t x} Q_{z}(\mathrm{~d} x)=\sum_{j=1}^{d} z_{j} H_{j}(t)$.

From the construction of the processes, it is clear that

$$
\psi_{0}(u)=\int_{A} \psi_{z}(u) v(\mathrm{~d} z)
$$


where $\psi_{z}$ is the ruin function for the one-dimensional model associated with $z \in A$. Using the bound in (14) together with the definition of $\kappa_{0}$, we obtain the Lundberg bound for the global ruin probabilities in the multivariate model. The same argument also shows that

$$
\limsup _{u \rightarrow \infty} \frac{1}{u} \log \psi_{0}(u) \leq-\kappa_{0} .
$$

To obtain a corresponding lower bound, we use the continuity of $z \mapsto \kappa(z)$ : there is a mixing vector $\bar{z}=\left(\bar{z}_{1}, \ldots, \bar{z}_{d}\right)$ in the compact set $A$ such that $\kappa_{0}=\kappa(\bar{z})$. For a given $\epsilon>0$, let $U$ be the intersection of an $\epsilon$-neighbourhood of $\bar{z}$ in $W_{d}$ with the support $A$ of $v$, and let $\tilde{z}=\left(\tilde{z}_{1}, \ldots, \tilde{z}_{d}\right)$ be defined by

$$
\tilde{z}_{j}:=\left(\bar{z}_{j}-\epsilon\right)^{+} \text {for } j=1, \ldots, d .
$$

With $\tilde{z}_{0}:=1-\|\tilde{z}\|$, we finally set

$$
\tilde{Q}:=\sum_{j=1}^{d} \tilde{z}_{j} Q_{j}+\tilde{z}_{0} \delta_{0}
$$

where $\delta_{0}$ denotes the one-point mass in 0 . We clearly have $\tilde{Q} \leq_{\text {st }} Q_{z}$, where ' $\leq_{\text {st }}$ ' means 'stochastically less than', in stochastic order for all $z \in U$, so that the ruin function $\tilde{\psi}$ for the one-dimensional model with premium income rate $\|c\|$, claim arrival intensity $\lambda$, and claim size distribution $\tilde{Q}$ provides a lower bound for $\psi_{z}$ for all $z \in U$. In particular,

$$
\psi_{0}(u) \geq v(U) \tilde{\psi}(u)
$$

so that, since $v(U)>0$ in view of $\bar{z} \in A$,

$$
\liminf _{u \rightarrow \infty} \frac{1}{u} \log \psi_{0}(u) \geq-\tilde{\kappa},
$$

where $\tilde{\kappa}$ denotes the Lundberg coefficient for the model with claim size distribution $\tilde{Q}$. Note that the tilded quantities depend on $\epsilon$ (whereas $\psi_{0}$ does not); hence, the proof will be complete if we can show that $\tilde{\kappa} \rightarrow \kappa_{0}$ as $\epsilon \rightarrow 0$.

Let $\tilde{H}$ be the moment generating function associated with $\tilde{Q}$, and let $J:=\left\{1 \leq j \leq d: \bar{z}_{j}>\right.$ $0\}$. Choose $\bar{t}$ such that $\kappa_{0}<\bar{t}<\min \left\{t_{0}^{j}: j \in J\right\}$. It is easy to see that

$$
H_{\bar{z}}(t)-\epsilon \sum_{j \in J} \sup _{0 \leq t \leq \bar{t}} H_{j}(t) \leq \tilde{H}(t) \leq H_{\bar{z}}(t)+\tilde{z}_{0} \quad \text { for all } t \in[0, \bar{t}] .
$$

As $H_{\bar{z}}$ is strictly convex and has a positive derivative in $\kappa_{0}$, these inequalities suffice to show that indeed $\tilde{\kappa} \rightarrow \kappa_{0}$ as $\epsilon \rightarrow 0$. This completes the proof of the theorem.

We may rephrase Theorem 7 by saying that, for mixed counting processes, the worst of the relevant rates for the corresponding one-dimensional models will be the rate for the multivariate model. It seems remarkable that only the support of $v$ matters, as far as the Lundberg bound and the Cramér asymptotics are concerned. Indeed, if $v$ has full support $W_{d}$ then the Lundberg exponent $\kappa_{0}$ for the multivariate model will simply be the minimum of the exponents $\kappa_{j}$ of the individual business lines. For example, for the model defined in (2), $v$ is determined by a $\operatorname{Beta}(1,1)$-distribution and $v$ has full support $W_{2}$. In this case $\kappa_{0}=\min \left\{\kappa_{1}, \kappa_{2}\right\}$.

We end this section by investigating the ruin probability for the components in the directionally mixed Poisson model separately. It may be helpful to compare the model with dependencies 
to a model where claims occur with the same marginal probabilities, but independently of each other. So suppose that $\left(X_{j}(n)\right)_{n \in \mathbb{N}_{0}}$ is the embedded process of component $j$ and $\left(X_{j}^{\perp}(n)\right)_{n \in \mathbb{N}_{0}}$ is the embedded process of the independent situation where claims arrive at average rate $\lambda \mathrm{E}\left[Z_{j}\right]$. In the example of the introduction where the transition probabilities are given by (2) this would mean that claims arrive with probability $\frac{1}{2}$ at jump times of $L$ and that $X_{1}^{\perp}(n)$ has a binomial $\mathrm{B}\left(n, \frac{1}{2}\right)$ distribution. A useful order to compare the risk of two random variables is the convex order (see, e.g. [13, p. 16]): let $\xi$ and $\eta$ be two random variables. We say that $\xi$ is smaller in the convex order than $\eta$, written $\xi \leq_{\mathrm{cx}} \eta$, if the inequality $\mathrm{E}[f(\xi)] \leq \mathrm{E}[f(\eta)]$ holds for all convex functions $f: \mathbb{R} \rightarrow \mathbb{R}$ for which the expectations are finite.

Theorem 8. If $N$ is a directionally mixed Poisson process with mixing distribution $v$ and base rate $\lambda$, then $X_{j}(n) \geq_{\mathrm{cx}} X_{j}^{\perp}(n)$ for all $n \in \mathbb{N}$ and $N_{j}(t) \geq_{\mathrm{cx}} N_{j}^{\perp}(t)$ for all $t \geq 0$.

Proof. By construction we have $X_{j}(n) \sim \mathrm{B}\left(n, Z_{j}\right)$ conditionally on $Z_{j}$ and $X_{j}^{\perp} \sim$ $\mathrm{B}\left(n, \mathrm{E}\left[Z_{j}\right]\right)$. Since $X_{j}(n)$ is a mixture of binomial distributions, a well-known theorem of de Finetti implies that $X_{j}(n) \geq_{\mathrm{cx}} X_{j}^{\perp}$ (see also [2, Theorem 4.1]). Since $N_{j}(t)=X_{j}(L(t)$ ) and the convex order is preserved under mixing (see [13, Example 2.5.3]), the proof is complete.

According to Theorem 3.1 of [12] this implies that the Lundberg exponent in the model with dependency-if it exists-is smaller than in the independent model. Now let us return to the special example with transition probabilities given by (2). In the corresponding independent case the Lundberg exponent exists if $c_{1}>\mu_{1} / 2$ and is given by the positive solution of $H(r)=1+2 c_{1} r$. However, this is a bad bound, as we will see. In this example the general considerations at the end of Subsection 5.3 can be applied and we obtain, for all $u \geq 0$,

$$
\psi_{1}(u) \geq \mathrm{P}\left[\frac{\delta}{\mu_{1}}+\frac{1}{2}<Z_{\infty}\right] .
$$

Hence, as $Z_{\infty}$ is uniformly distributed on the unit interval, $\delta$ has to be at least $\mu_{1} / 2$ in order to avoid the lower bound being strictly positive. In particular, if $c_{1}<\mu_{1}$, no Lundberg exponent exists! In this case calculating the premium by the expected value principle is very dangerous because just adding a little more than the expected cost may lead to a probability of ruin which cannot be controlled by increasing the initial reserve. If $\delta>\mu_{1} / 2$ or $c_{1}>\mu_{1}$, we obtain a Cramér asymptotic which can be computed with the help of Theorem 7 by setting $Q_{2}=\delta_{0}$ and $c_{2}=0$. In this case the Lundberg exponent exists and is given by the positive solution of $H(r)=1+c_{1} r$, which is of course smaller than in the independent case.

\section{Models with attracting intensities}

In the third of the examples given in Section 2 we have $d=2$ and intensities

$$
\beta_{1}\left(k_{1}, k_{2}\right)=\frac{1+k_{2}}{2+k_{1}+k_{2}}, \quad \beta_{2}\left(k_{1}, k_{2}\right)=\frac{1+k_{1}}{2+k_{1}+k_{2}} .
$$

From Theorem 4 we obtain, with $t \rightarrow \infty$,

$$
\frac{N_{1}(t)}{t} \stackrel{\mathrm{P}}{\rightarrow} \frac{1}{2}, \quad \frac{N_{1}(t)}{N_{2}(t)} \stackrel{\mathrm{P}}{\rightarrow} 1 .
$$

This means that in the long run both business lines generate the same number of claims, which of course is expected owing to the symmetric intensity. 
In this class an increasing proportion of claims of one type leads to an increasing rate of arrival of claims of the other type. The two business lines have a stabilising effect on each other. The corresponding limit function has the component functions

$$
\tilde{\beta}_{1}\left(x_{1}, x_{2}\right)=\frac{x_{2}}{x_{1}+x_{2}} \quad \text { and } \quad \tilde{\beta}_{2}\left(x_{1}, x_{2}\right)=\frac{x_{1}}{x_{1}+x_{2}} \quad \text { for all } x_{1}, x_{2} \in \mathbb{R}_{+}^{2} \backslash\{(0,0)\} .
$$

The conditions in Theorem 2 are satisfied, which means that every path of the fluid limit $\phi=\left(\phi_{1}, \phi_{2}\right)$ satisfies the differential equations

$$
\phi^{\prime}(t)=\tilde{\beta}(\phi(t)-\phi(0)) .
$$

Now, if we define $\rho_{i}(t):=\phi_{i}(t)-\phi_{i}(0)$, we obtain

$$
\rho_{1}^{\prime}=\frac{\rho_{2}}{\rho_{1}+\rho_{2}}, \quad \rho_{2}^{\prime}=\frac{\rho_{1}}{\rho_{1}+\rho_{2}} .
$$

This implies that $\rho_{1}^{\prime}+\rho_{2}^{\prime}=1$, and some straightforward manipulations lead to

$$
\rho_{1}(t)=\frac{1}{2}(a+t)+\frac{b}{a+t}, \quad \rho_{2}(t)=\frac{1}{2}(a+t)-\frac{b}{a+t} .
$$

Since we have the boundary condition $\rho_{1}(0)=\rho_{2}(0)=0$, it follows that $a=b=0$, and $\phi_{1}(t)=t / 2+\phi_{1}(0)$ and $\phi_{2}(t)=t / 2+\phi_{2}(0)$. Thus, the fluid limit turns out to be deterministic.

It is now interesting to investigate the embedded jump chain $(X(n))_{n \in \mathbb{N}_{0}}$ of $N$ in greater detail. From now on let us assume that $N_{1}(0)=N_{2}(0)=0$; in particular, we then have $X_{1}(n)+X_{2}(n)=n$ for all $n \in \mathbb{N}_{0}$. As in Section 6, there is a connection to an urn model: we begin with one red ball and one blue ball at time $n=0$, and in the successive steps we choose one of the balls uniformly at random and put it back into the urn, together with another ball of the opposite colour. If $\tilde{X}_{1}(n)$ and $\tilde{X}_{2}(n)$ denote the number of blue and red balls after $n$ steps, respectively, then we have $X_{i}(n)=\tilde{X}_{i}(n)-1$ for $i=1,2$ and all $n \in \mathbb{N}_{0}$. This leads to generalised Pólya-Eggenberger distributions, as discussed in [8, Chapter 4.3]. The recursions given there can be solved in the present special case, resulting in $\mathrm{E}\left[X_{1}(n)\right]=n / 2$, which is also clear owing to the symmetry, and $\operatorname{var}\left(X_{1}(n)\right)=(n+2) / 12$ for all $n \in \mathbb{N}$. Of course, $\operatorname{var}\left(X_{1}(0)\right)=0$. This can in turn be used in an obvious way to obtain $\mathrm{E}\left[N_{1}(t)\right]=t / 2$ and, by conditioning,

$$
\begin{aligned}
\operatorname{var}\left(N_{1}(t)\right) & =\operatorname{var}\left(\mathrm{E}\left[X_{1}\left(L_{t}\right) \mid L_{t}\right]\right)+\mathrm{E}\left[\operatorname{var}\left(X_{1}\left(L_{t}\right) \mid L_{t}\right)\right] \\
& =\frac{t}{3}+\frac{1}{6}\left(1-\mathrm{e}^{-t}\right)
\end{aligned}
$$

For the next result, we look at a more general model. We assume that $d=2$ and that $\left\|\beta\left(k_{1}, k_{2}\right)\right\|=1$ is constant for all $k \in E$. Moreover, we assume that

$$
\beta_{1}\left(k_{1}, k_{2}\right) \leq \frac{1}{2} \quad \text { if } k_{1} \geq k_{2}, \quad \beta_{1}\left(k_{1}, k_{2}\right) \geq \frac{1}{2} \quad \text { if } k_{1} \leq k_{2} .
$$

This models a situation where there is a tendency towards the diagonal, which includes cases such as (3). We want to compare $\left(X_{1}(n)\right)_{n \in \mathbb{N}_{0}}$ with a sequence of random variables $\left(X_{1}^{\perp}(n)\right)_{n \in \mathbb{N}_{0}}$ where claims arrive independently of each other with rate $\frac{1}{2}$. Thus, $X_{1}^{\perp}(0):=0$ and, for $n \in \mathbb{N}$,

$$
X_{1}^{\perp}(n):=\sum_{k=1}^{n} I_{k}
$$


where $\left(I_{n}\right)_{n \in \mathbb{N}}$ is a sequence of independent and identically distributed random variables with $\mathrm{P}\left[I_{1}=1\right]=\mathrm{P}\left[I_{1}=0\right]=\frac{1}{2}$, i.e. $X_{1}^{\perp}(n)$ has a binomial $\mathrm{B}\left(n, \frac{1}{2}\right)$ distribution. Let $X_{2}^{\perp}(n):=$ $n-X_{1}^{\perp}(n)$, and denote by $N^{(1 / 2)}$ a Poisson process with intensity $\frac{1}{2}$.

Theorem 9. Under the preceding conditions, we have $X_{1}(n) \leq_{\mathrm{cx}} X_{1}^{\perp}(n)$ for all $n \in \mathbb{N}$ and $N_{1}(t) \leq_{\mathrm{cx}} N^{(1 / 2)}(t)$ for all $t \geq 0$.

Proof. Let

$$
Y_{m}(n):=\min \left\{X_{1}(n), X_{2}(n)\right\}, \quad Y_{M}(n):=\max \left\{X_{1}(n), X_{2}(n)\right\},
$$

and analogously let

$$
Y_{m}^{\perp}(n):=\min \left\{X_{1}^{\perp}(n), X_{2}^{\perp}(n)\right\}, \quad Y_{M}^{\perp}(n):=\max \left\{X_{1}^{\perp}(n), X_{2}^{\perp}(n)\right\} .
$$

We suppose that all these sequences are generated by the same sequence $\left(U_{n}\right)_{n \in \mathbb{N}_{0}}$ of independent and identically distributed, uniformly over the interval $(0,1)$, random variables, as follows (where we suppose, without loss of generality, that $Y_{m}^{\perp}(n)=X_{1}^{\perp}(n)$ and $Y_{m}(n)=X_{1}(n)$ ):

$$
X_{1}(n+1):=X_{1}(n)+\mathbf{1}_{\left[U_{n} \leq \beta_{1}\left(X_{1}(n), X_{2}(n)\right)\right]} \quad \text { and } \quad X_{1}^{\perp}(n+1):=X_{1}^{\perp}(n)+\mathbf{1}_{\left[U_{n} \leq 1 / 2\right]} .
$$

Via an induction over $n$, we can show that, for all $n \in \mathbb{N}$,

$$
Y_{m}^{\perp}(n) \leq Y_{m}(n) \leq Y_{M}(n) \leq Y_{M}^{\perp}(n) .
$$

The proof is as follows. For $n=1$, we have $X_{1}(1)=\mathbf{1}_{\left[U_{0} \leq 1 / 2\right]}=X_{1}^{\perp}(1)$ and the inequality is true. Now suppose that the inequality holds for $k=1, \ldots, n$ and suppose, without loss of generality, that $Y_{m}^{\perp}(n)=X_{1}^{\perp}(n), Y_{m}(n)=X_{1}(n)$, and $Y_{m}(n)<Y_{M}(n)$. In this case we must have $X_{1}(n) \leq n / 2$, which implies that $\beta_{1}\left(X_{1}(n), X_{2}(n)\right) \geq \frac{1}{2}$. Hence,

$$
\mathbf{1}_{\left[U_{n} \leq 1 / 2\right]} \leq \mathbf{1}_{\left[U_{n} \leq \beta_{1}\left(X_{1}(n), X_{2}(n)\right)\right]},
$$

and, thus,

$$
X_{1}^{\perp}(n+1) \leq X_{1}(n+1) \leq X_{2}(n+1) \leq X_{2}^{\perp}(n+1) .
$$

In the case in which $Y_{m}(n)=Y_{M}(n)$ we must have $X_{1}(n)=X_{2}(n)=n / 2$. If also $X_{1}^{\perp}(n)=$ $X_{2}^{\perp}(n)=n / 2$, the induction step is trivial. If

$$
X_{1}^{\perp}(n)<X_{1}(n)=X_{2}(n)<X_{2}^{\perp}(n),
$$

the inequality at time $n+1$ remains valid no matter what happens and (17) is proved.

Next let $f: \mathbb{R} \rightarrow \mathbb{R}$ be a convex function. Thus, we obtain (note that the sum of the respective minimum and maximum is always $n$ )

$$
f\left(Y_{M}^{\perp}(n)\right)+f\left(Y_{m}^{\perp}(n)\right) \geq f\left(Y_{M}(n)\right)+f\left(Y_{m}(n)\right) .
$$

But this yields

$$
\begin{aligned}
\mathrm{E}\left[f\left(X_{1}(n)\right)\right] & =\frac{1}{2} \mathrm{E}\left[f\left(Y_{m}(n)\right)\right]+\frac{1}{2} \mathrm{E}\left[f\left(Y_{M}(n)\right)\right] \\
& \leq \frac{1}{2} \mathrm{E}\left[f\left(Y_{m}^{\perp}(n)\right)\right]+\frac{1}{2} \mathrm{E}\left[f\left(Y_{M}^{\perp}(n)\right)\right] \\
& =\mathrm{E}\left[f\left(X_{1}^{\perp}(n)\right)\right],
\end{aligned}
$$

and, hence, the first assertion is proved. Moreover, $N_{1}(t)=X_{1}(L(t))$ and $N^{(1 / 2)}(t)=$ $X_{1}^{\perp}(L(t))$, where $L$ is a Poisson process with intensity 1 . Since the convex order is preserved under mixing (see [13, Example 2.5.3]), the proof is complete. 
Next we derive Lundberg exponents for the global ruin probability $\psi_{0}$ as in Section 6 for the interacting intensity models with intensities satisfying (16). We assume an initial capital $u_{j}$, a premium income rate $c_{j}$, and a claim size distribution $Q_{j}$ for the $j$ th business line, $j=1,2$. As before, $\mu_{j}:=\int x Q_{j}(\mathrm{~d} x)<\infty$. Moreover, we define $Q^{*}:=\frac{1}{2} Q_{1}+\frac{1}{2} Q_{2}$, and denote by $H^{*}(r):=\int \mathrm{e}^{r x} Q^{*}(\mathrm{~d} x)$ the moment generating function of $Q^{*}$.

Theorem 10. Suppose that $\beta_{1}\left(X_{1}(n), X_{2}(n)\right) \stackrel{\mathrm{P}}{\rightarrow} \frac{1}{2}$ for $n \rightarrow \infty,\|c\|>\left(\mu_{1}+\mu_{2}\right) / 2$, and that $Q_{1} \leq_{\text {st }} Q_{2}$ or $Q_{2} \leq_{\text {st }} Q_{1}$. Then it holds that

$$
\lim _{u \rightarrow \infty} \frac{1}{u} \log \psi_{0}(u)=-\kappa,
$$

where $\kappa$ is the positive solution of the equation $H^{*}(r)=1+\|c\| r$.

Proof. Let us look at a discrete skeleton of $\|R(t)\|$. Let $T_{1}, T_{2}, \ldots$ be a sequence of independent and identically $\operatorname{Exp}(1)$-distributed random variables and define, for $n \in \mathbb{N}$,

$$
Y_{n}:=\sum_{k=1}^{X_{1}(n)} U_{1, k}+\sum_{k=1}^{n-X_{1}(n)} U_{2, k}-\|c\| \sum_{k=1}^{n} T_{k},
$$

where $X_{1}(n)$ is as usual the embedded chain, and $U_{1, k} \sim Q_{1}$ and $U_{2, k} \sim Q_{2}$. Obviously, we have $\psi_{0}(u)=\mathrm{P}$ (there exists $\left.n \in \mathbb{N}, Y_{n}>u\right)$. Now let

$$
Y_{n}^{\perp}:=\sum_{k=1}^{n} U_{k}-\|c\| \sum_{k=1}^{n} T_{k},
$$

where $U_{k} \sim Q^{*}=\frac{1}{2} Q_{1}+\frac{1}{2} Q_{2}$. We now claim that, for all $n \in \mathbb{N}$,

$$
Y_{n} \leq_{\mathrm{cx}} Y_{n}^{\perp} \text {. }
$$

Obviously, the expectations are the same on both sides and, owing to the convolution property of the convex order and Theorem 9, it suffices to show that

$$
\pi_{t}(l):=\mathrm{E}\left[\sum_{k=1}^{l} U_{1, k}+\sum_{k=1}^{n-l} U_{2, k}-t\right]_{+}
$$

is convex (see also [13, Theorem 4.3.6]). This follows from the following inequality, where we assume that $Q_{1} \geq_{\text {st }} Q_{2}$ :

$$
\begin{aligned}
\pi_{t}(l+1)-\pi_{t}(l)= & \mathrm{E}\left[\sum_{k=1}^{l-1} U_{1, k}+\sum_{k=1}^{n-l-1} U_{2, k}+U_{1, l}+U_{1, l+1}-t\right]_{+} \\
& -\mathrm{E}\left[\sum_{k=1}^{l-1} U_{1, k}+\sum_{k=1}^{n-l-1} U_{2, k}+U_{1, l}+U_{2, n-l}-t\right]_{+} \\
\geq & \mathrm{E}\left[\sum_{k=1}^{l-1} U_{1, k}+\sum_{k=1}^{n-l-1} U_{2, k}+U_{2, n-l+1}+U_{1, l+1}-t\right]_{+} \\
& -\mathrm{E}\left[\sum_{k=1}^{l-1} U_{1, k}+\sum_{k=1}^{n-l-1} U_{2, k}+U_{2, n-l+1}+U_{2, n-l}-t\right]_{+} \\
= & \pi_{t}(l)-\pi_{t}(l-1) .
\end{aligned}
$$


Thus, the statement is shown and it follows that $\mathrm{E}\left[\exp \left(r Y_{n}\right)\right] \leq \mathrm{E}\left[\exp \left(r Y_{n}^{\perp}\right)\right]$ for all $n \in \mathbb{N}$ and $r \in \mathbb{R}$. A simple calculation shows that

$$
\mathrm{E}\left[\exp \left(r Y_{n}^{\perp}\right)\right]=\left(\frac{H^{*}(r)}{1+\|c\| r}\right)^{n}
$$

and

$$
g(r):=\lim _{n \rightarrow \infty} \frac{1}{n} \log \left(\mathrm{E}\left[\exp \left(r Y_{n}^{\perp}\right)\right]\right)=\log \left(\frac{H^{*}(r)}{1+\|c\| r}\right) .
$$

We can see that $g(r)=0$ if and only if $H^{*}(r)=1+\|c\| r$. Since $H^{*}(r)=\left(H_{1}(r)+H_{2}(r)\right) / 2$, this equation has a strictly positive solution under our assumption $\|c\|>\left(\mu_{1}+\mu_{2}\right) / 2$. On the other hand, we have, with

$$
A_{k}:=\left\{\omega:\left|\beta_{1}\left(X_{1}(n), X_{2}(n)\right)-\frac{1}{2}\right| \leq \varepsilon \text { for all } n \geq k\right\},
$$

(note that $\mathrm{P}\left[A_{k}\right] \rightarrow 1$ for $k \rightarrow \infty$ on a suitably defined probability space, owing to Skorokhod's representation theorem)

$$
\begin{aligned}
\mathrm{E}\left[\exp \left(r Y_{n}\right)\right] & =\mathrm{E}\left[\exp \left(r Y_{n}\right) \mid A_{k}\right] \mathrm{P}\left[A_{k}\right]+\mathrm{E}\left[\exp \left(r Y_{n}\right) \mid A_{k}^{\mathrm{c}}\right] \mathrm{P}\left[A_{k}^{\mathrm{c}}\right] \\
& \geq \mathrm{E}\left[\exp \left(r Y_{n}\right) \mid A_{k}\right] \mathrm{P}\left[A_{k}\right] \\
& \geq \mathrm{E}\left[\exp \left(r Y_{n}^{\varepsilon}\right)\right] \mathrm{P}\left[A_{k}\right],
\end{aligned}
$$

where we have written $A^{\mathrm{c}}$ for the complement of a set $A$ and, for $n \geq k$,

$$
Y_{n}^{\varepsilon}=\sum_{j=1}^{X_{1}^{\varepsilon}(n)} U_{1, j}+\sum_{j=1}^{X_{2}^{\varepsilon}(n)} U_{2, j}-\|c\| \sum_{i=1}^{n} T_{i}
$$

and $\left(X_{1}^{\varepsilon}(n), X_{2}^{\varepsilon}(n), n-X_{1}^{\varepsilon}(n)-X_{2}^{\varepsilon}(n)\right) \sim M\left(n-k, \frac{1}{2}-\varepsilon, \frac{1}{2}-\varepsilon, 2 \varepsilon\right)$ has a multinomial distribution. We obtain

$$
g_{\varepsilon}(r):=\lim _{n \rightarrow \infty} \frac{1}{n} \log \left(\mathrm{E}\left[\exp \left(r Y_{n}^{\varepsilon}\right)\right]\right)=\log \left(\frac{1}{1+\|c\| r}\left(\left(\frac{1}{2}-\varepsilon\right) H_{1}(r)+\left(\frac{1}{2}-\varepsilon\right) H_{2}(r)\right)\right) .
$$

Thus, whenever the limit exists, we have, for all $\varepsilon>0$,

$$
g_{\varepsilon}(r) \leq \lim _{n \rightarrow \infty} \frac{1}{n} \log \left(\mathrm{E}\left[\exp \left(r Y_{n}\right)\right]\right) \leq g(r)
$$

Letting $\varepsilon \downarrow 0$, we obtain

$$
\lim _{n \rightarrow \infty} \frac{1}{n} \log \left(\mathrm{E}\left[\exp \left(r Y_{n}\right)\right]\right)=g(r)
$$

The statement now follows from Theorem 3.1 of [12].

The above proof is based on the asymptotics of the moment generating functions of a suitably chosen sequence of random variables embedded into the risk process. The underlying application of the Gärtner-Ellis theorem was introduced in the closely related queueing context by Glynn and Whitt [7].

Obviously, we find here that the Lundberg exponent is the same as in the case where the claims occur independently with probability $\frac{1}{2}$. Also, note that the convergence condition $\beta_{1}\left(X_{1}(n), X_{2}(n)\right) \stackrel{\mathrm{P}}{\rightarrow} \frac{1}{2}$ is satisfied in the example where $\beta$ is given by (3), owing to Theorem 4 . If we consider the business lines separately, we obtain a Cramér asymptotic if $c_{1}>\mu_{1} / 2$ and the Lundberg exponent is given by the positive solution of $H_{1}(r)=1+2 c_{1} r$. 


\section{References}

[1] BäUerle, N. AND GrüBel, R. (2005). Multivariate counting processes: copulas and beyond. ASTIN Bull. 35, 379-408.

[2] BäUerle, N. AND Müller, A. (1998). Modeling and comparing dependencies in multivariate risk portfolios. ASTIN Bull. 28, 59-76.

[3] Bäuerle, N., Müller, A. And Blatter, A. (2008). Dependence properties and comparison results for Lévy processes. Math. Meth. Operat. Res. 67, 161-186.

[4] Cont, R. And Tankov, P. (2004). Financial Modelling with Jump Processes. Chapman \& Hall/CRC, Boca Raton, FL.

[5] Davis, M. H. A. (1993). Markov Models and Optimization (Monogr. Statist. Appl. Prob. 49). Chapman \& Hall, London.

[6] Feller, W. (1971). An Introduction to Probability Theory and Its Applications, Vol. II, 2nd edn. John Wiley, New York.

[7] Glynn, P. W. and Whitt, W. (1994). Logarithmic asymptotics for steady-state tail probabilities in a singleserver queue. In Studies in Applied Probability (J. Appl. Prob. Spec. Vol. 31A), Applied Probability Trust, Sheffield, pp. 131-156.

[8] Johnson, N. L. And Kotz, S. (1977). Urn Models and Their Application. John Wiley, New York.

[9] Kemeny, J. G., Snell, J. L. And Knapp, A. W. (1976). Denumerable Markov Chains, 2nd edn. Springer, New York.

[10] Kushner, H. J. And Dupuis, P. (2001). Numerical Methods for Stochastic Control Problems in Continuous Time, 2nd edn. Springer, New York.

[11] Liggett, T. M. (1985). Interacting Particle Systems. Springer, New York.

[12] Müller, A. ANd Pflug, G. (2001). Asymptotic ruin probabilities for dependent claims. Insurance Math. Econom. 28, 381-392.

[13] Müller, A. And Stoyan, D. (2002). Comparison Methods for Stochastic Models and Risks. John Wiley, Chichester.

[14] Pfeifer, D. AND NeŠLehovÁ, J. (2004). Modeling and generating dependent risk processes for IRM and DFA. ASTIN Bull. 34, 333-360.

[15] Rolski, T., Schmidli, H., Schmidt, V. And Teugels, J. (1999). Stochastic Processes for Insurance and Finance. John Wiley, Chichester.

[16] Sawyer, S. A. (1997). Martin boundaries and random walks. In Harmonic Functions on Trees and Buildings (New York, 1995; Contemp. Math. 206), American Mathematical Society, Providence, RI, pp. 17-44.

[17] Szekli, R. (1995). Stochastic Ordering and Dependence in Applied Probability (Lecture Notes Statist. 97). Springer, New York.

[18] Whitt, W. (2002). Stochastic-Process Limits. An Introduction to Stochastic-Process Limits and Their Application to Queues. Springer, New York.

[19] Zocher, M. (2003). Multivariate mixed Poisson processes and the dependence of their coordinates. Dresdner Schriften zur Versicherungsmathematik 2, 1-6.

[20] Zocher, M. (2005). Multivariate mixed Poisson processes. Doctoral Thesis, Technische Universität Dresden. 